\title{
Supporting Information: Silver Nanodisk Monolayers with Surface Coverage Gradients for Use as Optical Rulers and Protractors
}

\author{
Mahmoud A. Mahmoud* \\ Laser Dynamics Laboratory, School of Chemistry and Biochemistry, Georgia Institute of Technology, \\ Atlanta, Georgia 30332-0400 \\ *E-mail: $\underline{\text { mmahmoud@gatech.edu }}$
}




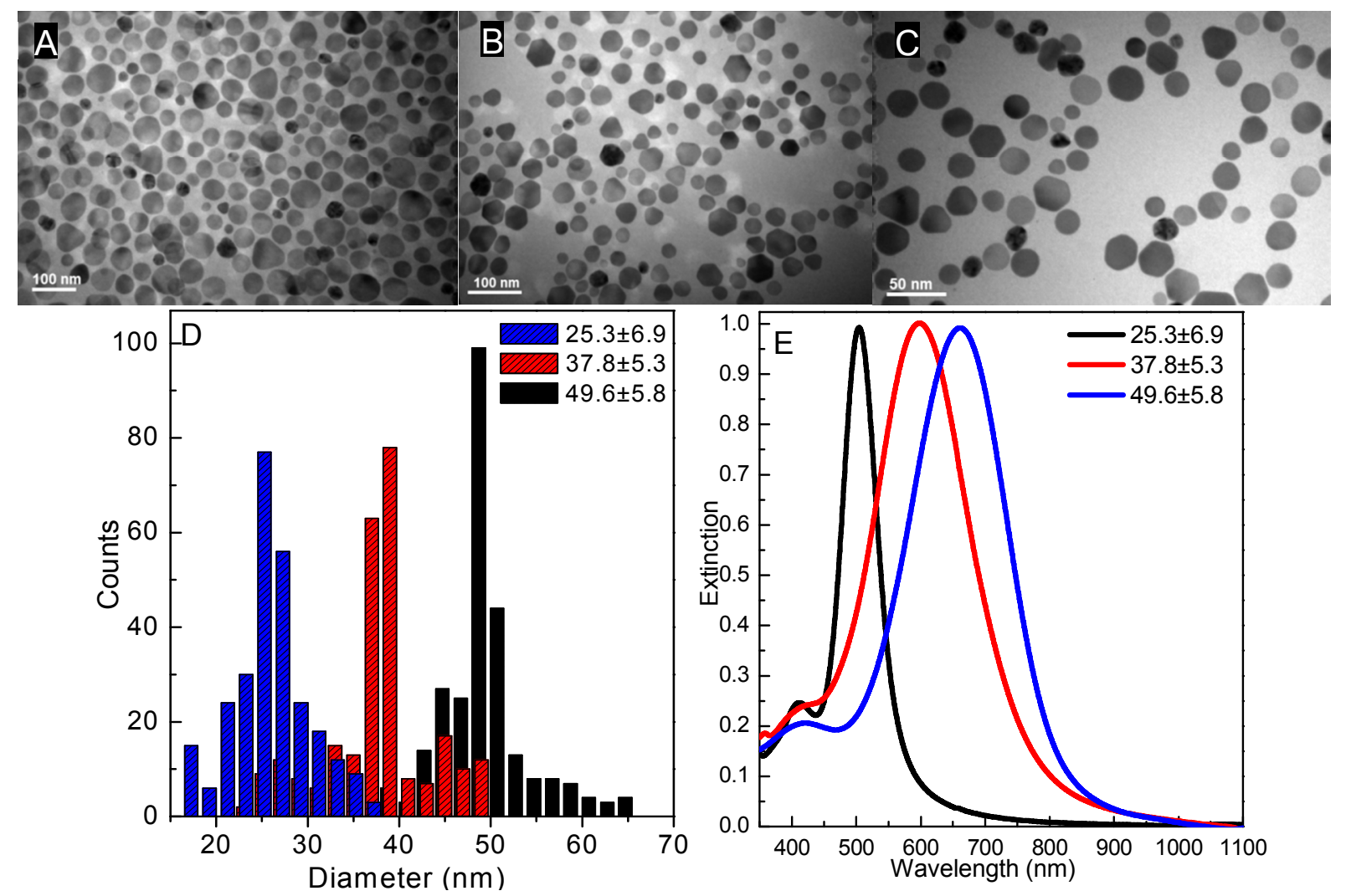

Figure S1 TEM image of the as prepared silver nanodisks of average diameter of: A) $50 \mathrm{~nm}, \mathrm{~B}$ ) $38 \mathrm{~nm}$, and $25 \mathrm{~nm}$. D) Statistical analysis of the diameters of AgNDs, ImageJ was used to determine the diameters of the AgNDs. E) LSPR spectra of the AgNDs dispersed in water.

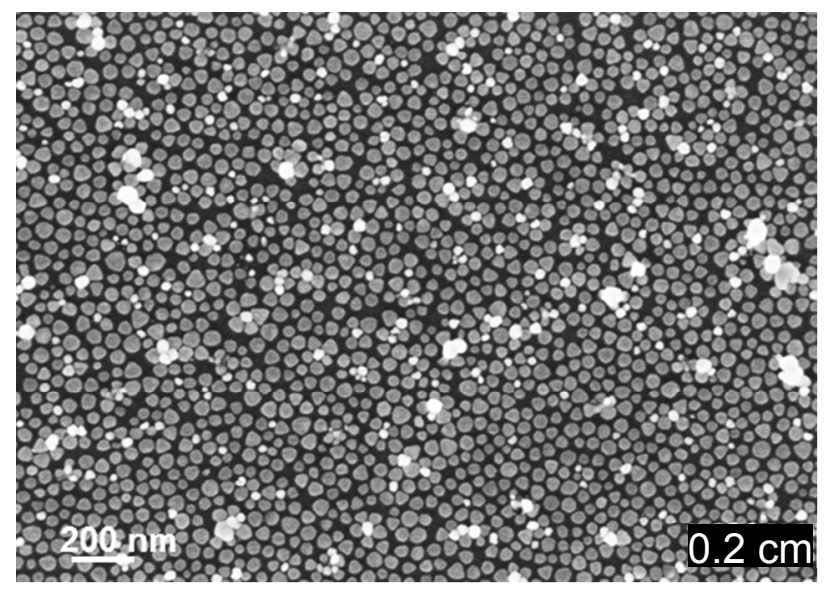

Figure S2 SEM image of monolayer assembly of $50 \mathrm{~nm}$ AgND conducted at $0.2 \mathrm{~cm}$ separation from the highest coverage spot. 

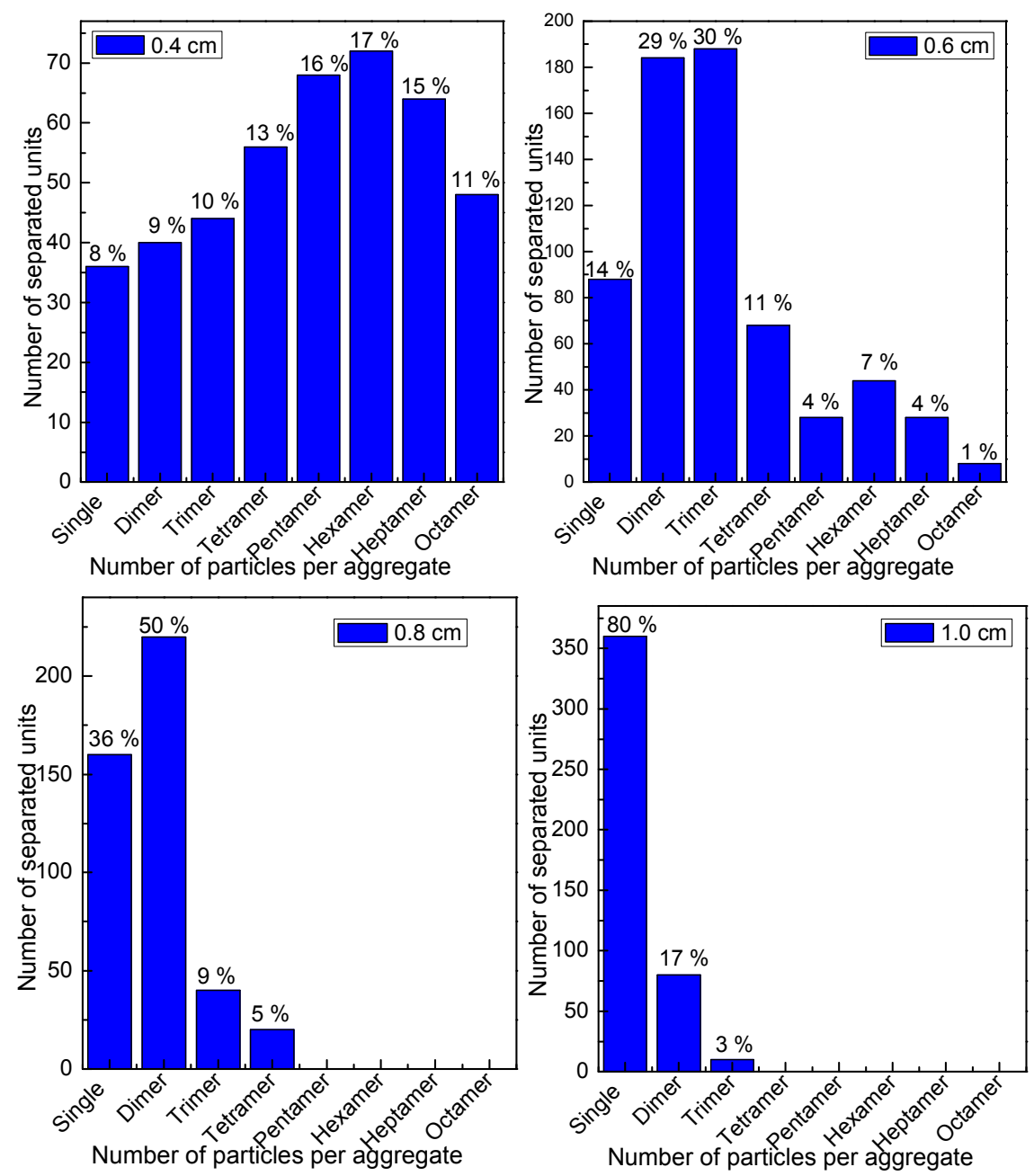

Figure S3 Statistical analysis for the separated units in the SEM images either single AgND or separated groups of aggregates. 


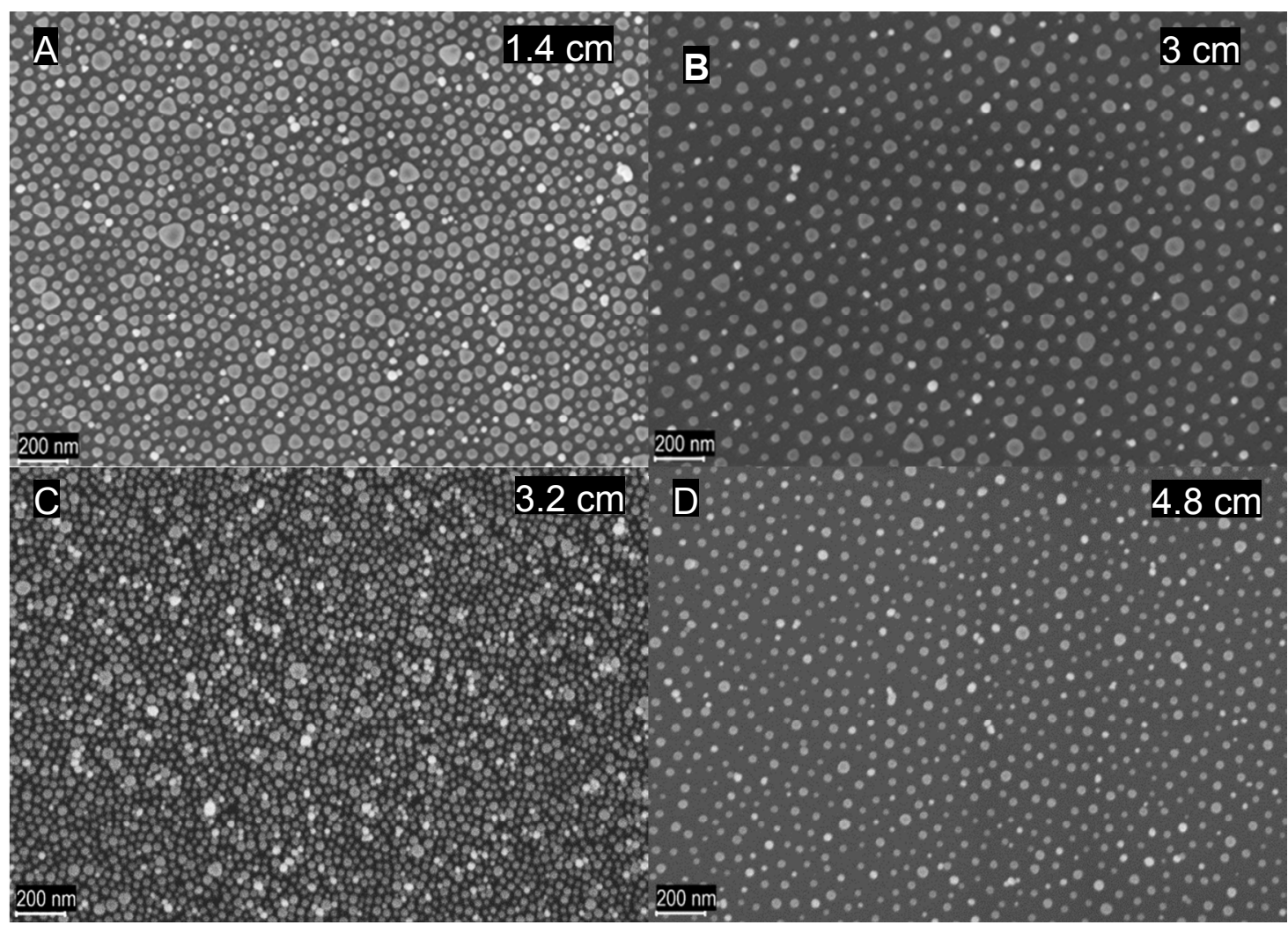

Figure S4 SEM image of monolayer assembly of: $38 \mathrm{~nm} \mathrm{AgND} \mathrm{conducted} \mathrm{at} \mathrm{A)} 1.4 \mathrm{~cm}$ and B) 3 $\mathrm{cm}$ separation from the highest coverage spot, AgNDs of $25 \mathrm{~nm}$ diameter for C) $3.2 \mathrm{~cm}$ spot and D) 4.8 spot.

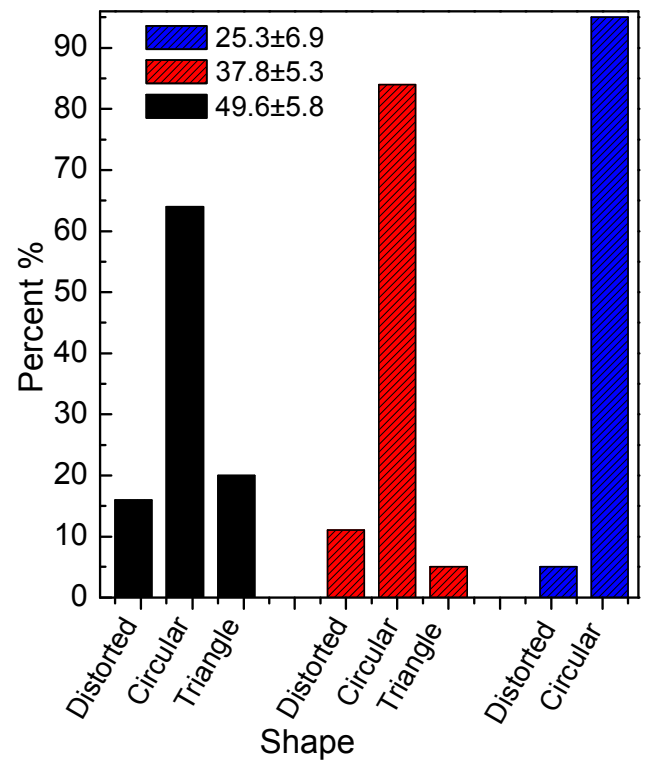

Figure S5 Statistical analysis of the shapes of AgNDs of different diameters. Three different shapes are observed: circular, triangular, and distorted (any other shape). The statistical analysis of the shape is based on the SEM images. 

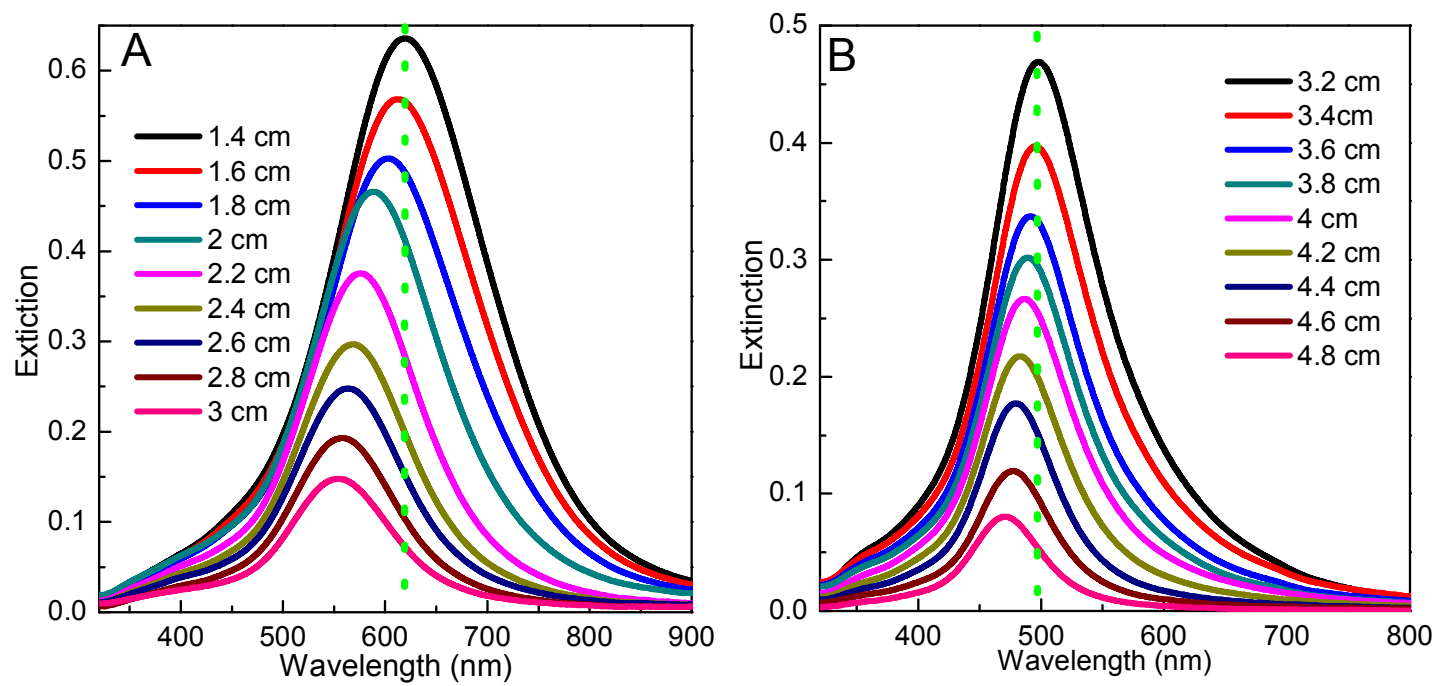

Figures S6 LSPR spectrum of the CDG AgND monolayer of diameter of: A) $38 \mathrm{~nm}$ and B) 25 $\mathrm{nm}$ measured at different separation distance from the HCS of the CDG $50 \mathrm{~nm}$ AgNDs.
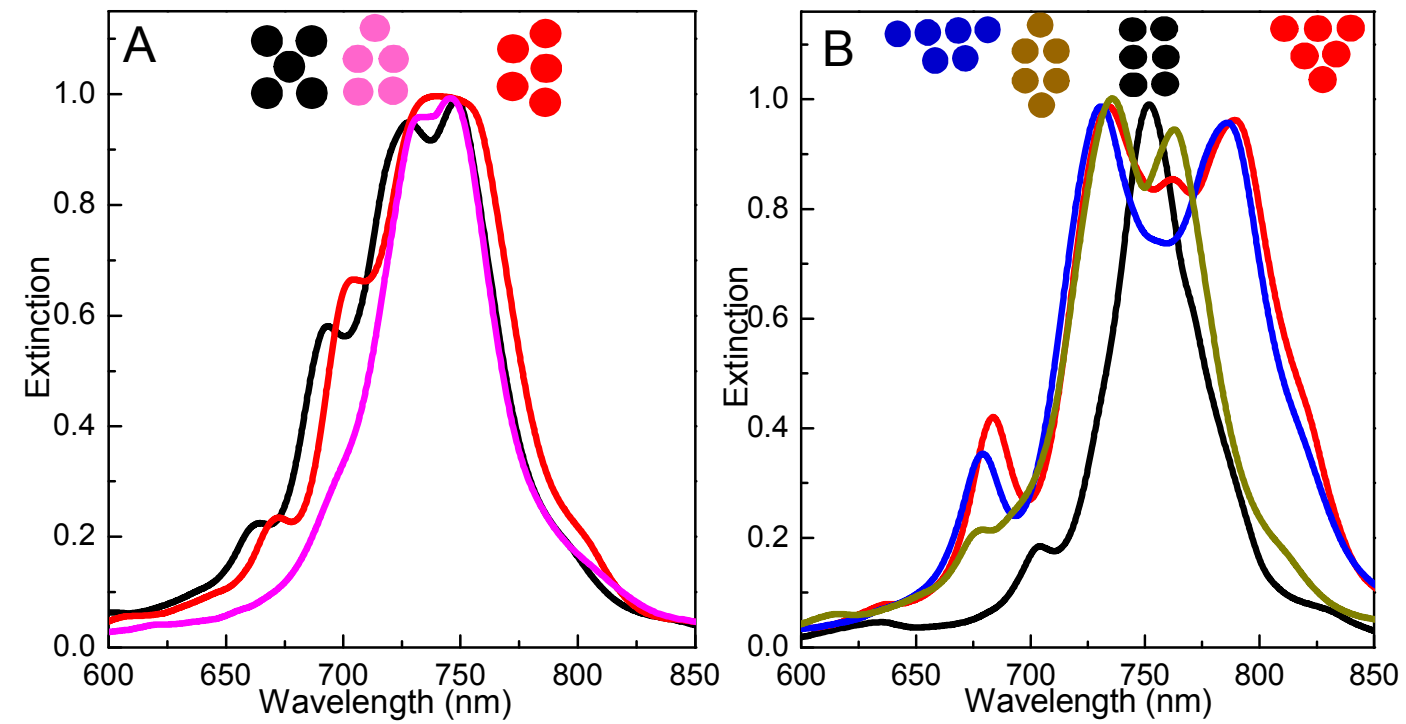

Figure S7 Simulated DDA LSPR spectra of an AgND pentamer (A) and hexamer (B) with different configurations. 

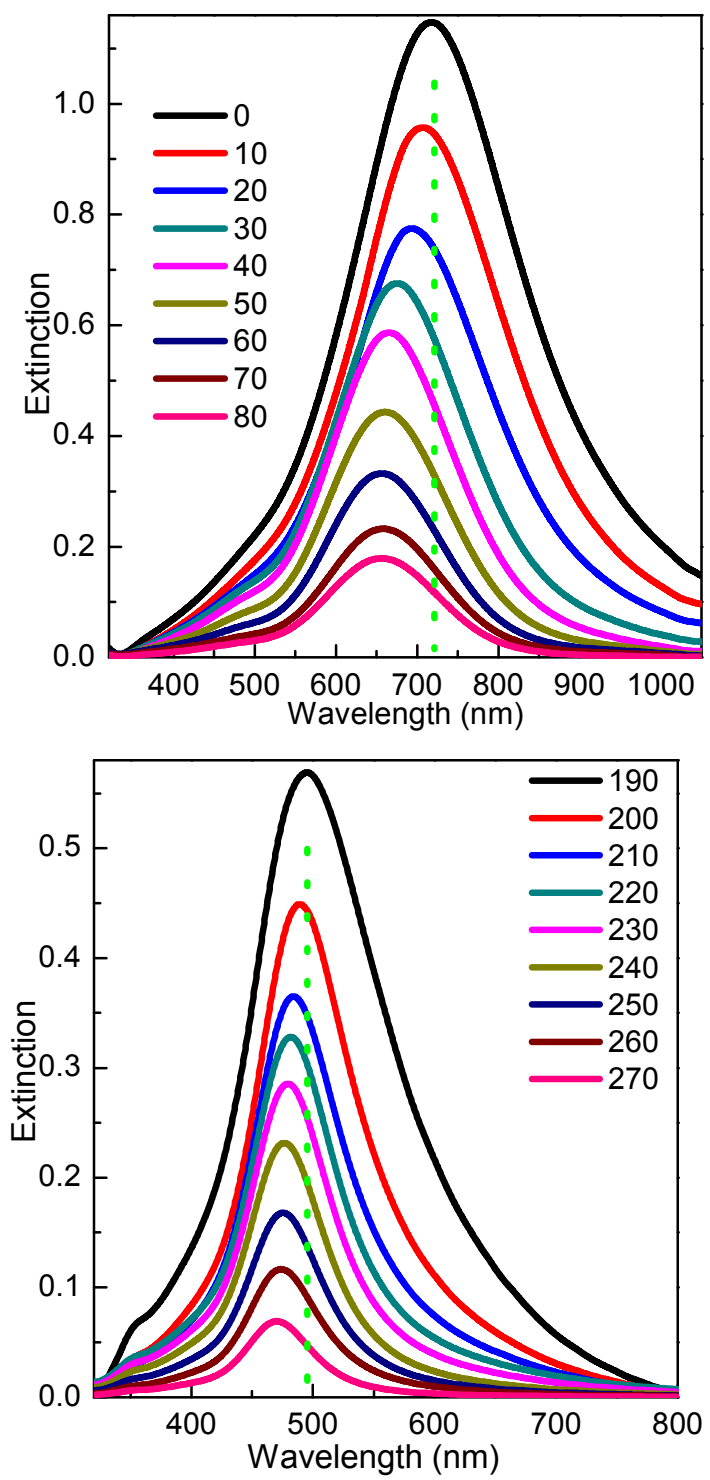

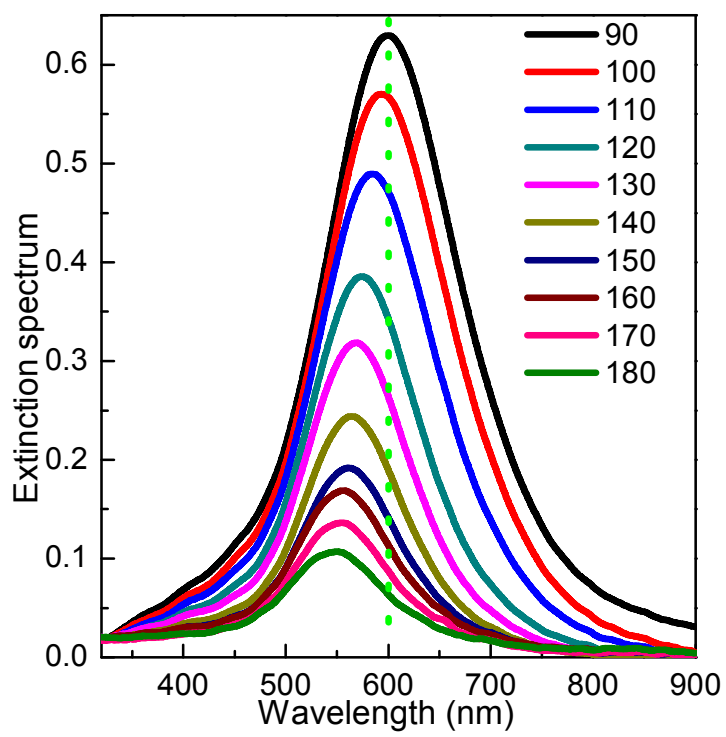

Figure S8 the LSPR spectrum of: A) $50 \mathrm{~nm}$ AgND CDG on circular substrate measured at different angle of rotation starting from the HCS " $0^{\circ}$ " up to $80^{\circ}$, B) $38 \mathrm{~nm} \mathrm{AgNDs}$ collected when the angle of rotation was change between $90^{\circ}$ to $180^{\circ}$, C) $25 \mathrm{~nm}$ $\mathrm{AgNCs}$ obtained when the circular quartz substrate when the angle of rotation was increased from $190^{\circ}$ to $270^{\circ}$. 